\title{
LEGO as Language for Visual Communication
}

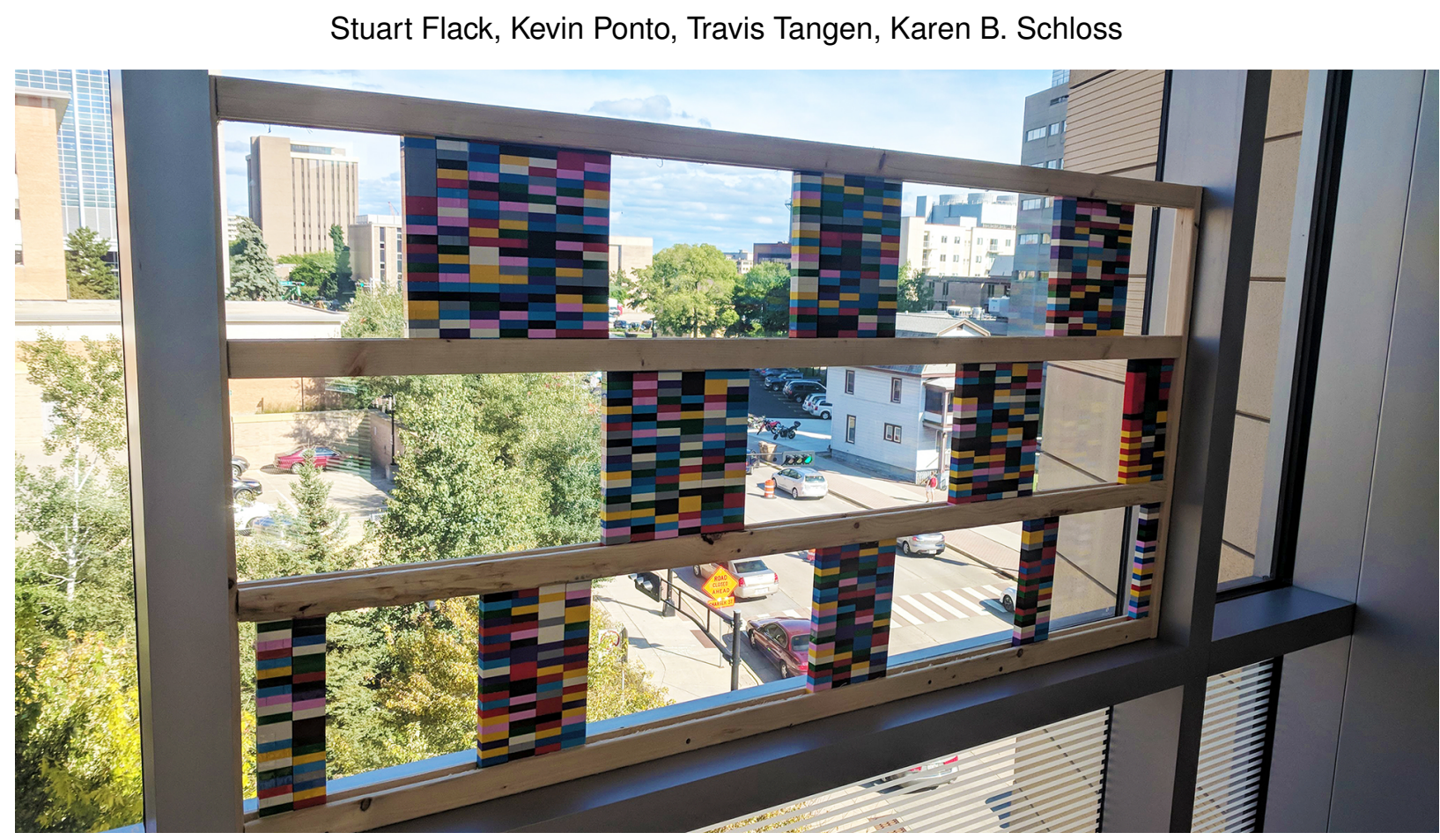

Figure 1. Data generated from the "What color is _-_-_?" project. Participants built stacks of LEGO to encode data about themselves and their surroundings during a community event at the University of Wisconsin-Madison, Discovery Building. Each stack was assembled by a different participant or family, and each row represents an answer to a different question (see Figure 2).

\begin{abstract}
We are using LEGO for a variety of new tasks like surveys, data capture, and data visualization. We have found that LEGO is a low-tech high-touch approach to mapping and data visualization. Through two projects we explore how standard LEGO sets can be used with both children and adults to gather information, present it in an appealing way, and catalyze memorable conversations about that information in community based settings. The first project, "What Color is ____?" used LEGO to conduct and visualize answers to a 21 question survey with children and families at the 2018 Wisconsin Science Festival. The second project uses LEGO to visualize citizen science data on air quality on the South and Westside of Chicago. We believe that using LEGO for data collection and visualization will enable new forms of fact driven community based advocacy.
\end{abstract}

Index Terms-Visual Communication, Information Visualization, Data Visualization, Visual Reasoning, Manipulatives, Survey Techniques, Data Visualization, Community Advocacy, Air Quality, Environmental Justice

\section{INTRODUCTION}

Over 400 billion LEGO have been manufactured and sold since the Danish company began keeping track in 1958. The colorful, interlocking blocks are now globally ubiquitous. They are widely used in homes, schools, and museums, for sculpture, construction, robotics, creative play, and gouging the bare feet of unsuspecting parents in dark bedrooms. Yet, despite the intensive use of LEGO for over 60 years, there is still much to explore in LEGO's potential as a learning and thinking tool.

We are using LEGO as a medium for conducting surveys by cap-

- Stuart Flack, Invisible Institute and Environmental Law Policy Center. Email: flackstuart@gmail.com.

- Kevin Ponto, Department of Design Studies and Wisconsin Institute for Discovery, University of Wisconisn-Madison,Email: kbponto@wisc.edu.

- Travis Tangen, Discovery Outreach, Wisconsin Alumni Research Foundation. Email: ttangen@warf.org.

- Karen B. Schloss, Department of Psychology and Wisconsin Institute for Discovery, University of Wisconsin-Madison.Email: kschloss@wisc.edu. turing, storing, and visualizing information in LEGO structures. Our recent projects at the Wisconsin Science Festival and citizen science efforts with The Environmental Law \& Policy and The Invisible Institute in Chicago's neighborhoods show that LEGO can be deployed for tasks of data capture and visualization. Simultaneously, we are beginning to use LEGO to catalyze community discussion and action around data. We believe that the process of assembling LEGO into tangible three-dimensional, multi-color visualizations allows people to experience data about their world in unique and delightful new ways. In turn, this opens new possibilities for understanding, advocacy, and social change. In the following sections we first motivate our approach for using LEGO for data collection and visualization. We then describe two projects that deploy our methods with the general public during community events.

\section{WHY LEGO?}

LEGO has exciting potential to engage the public in data visualization and discourse for several key reasons, which we outline below. 

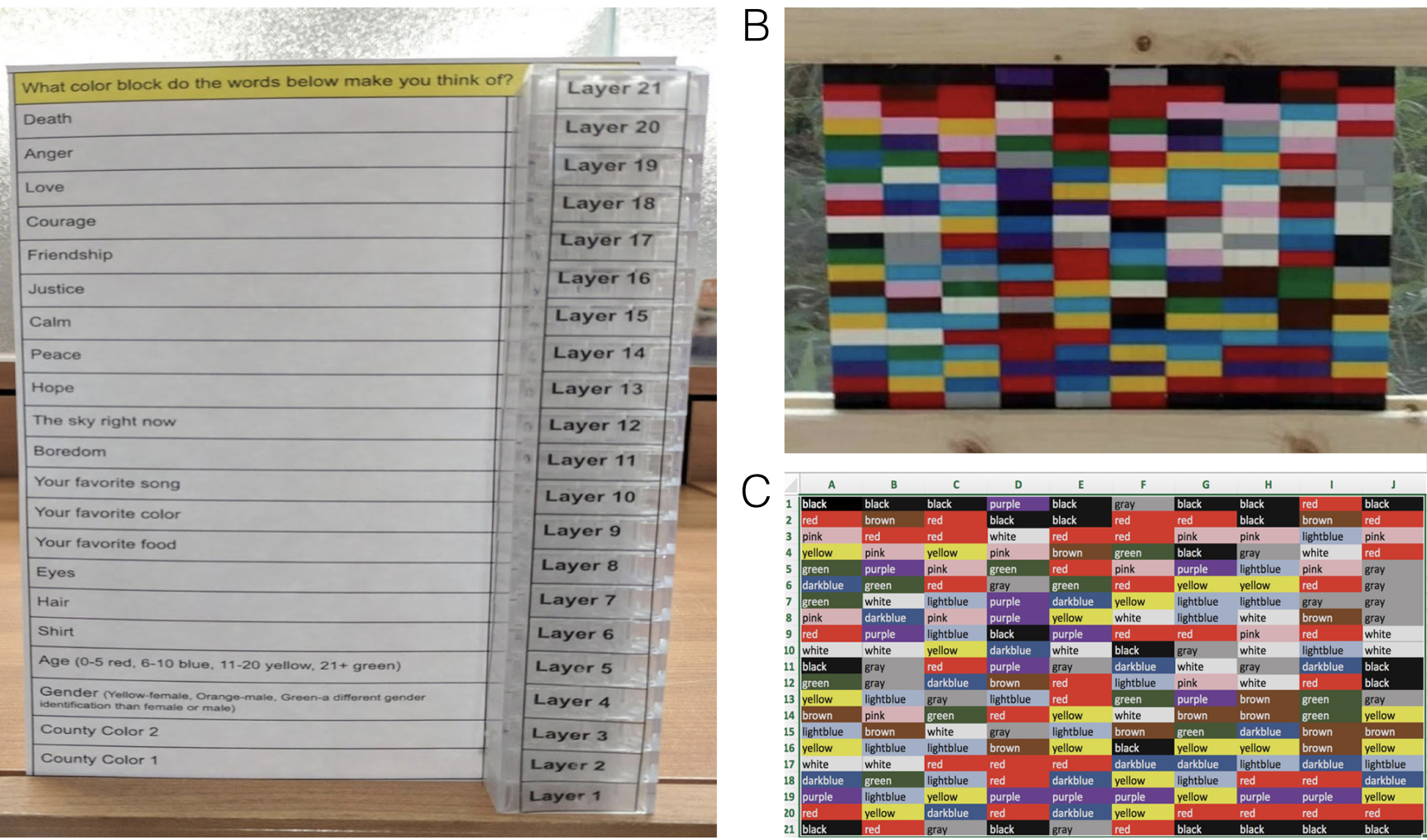

Figure 2. A. List of questions and template that participants used to build their LEGO stacks in "What Color is _-__?". B. Photograph of a block of LEGO stacks constructed by participants. C. Spreadsheet produced after imaging the LEGO stacks from B and automatically digitizing the data.

\subsection{LEGO are fun}

Constructing and deconstructing LEGO structures has captured the interest of children for generations. Through sound, color, shape, and texture, LEGO draw on multiple senses and engage us in a unique way that is similar to play, contrasting with conventional survey approaches As a result, LEGO surveys are inherently engaging. We are breaking barriers between science and play by building data visualization into this household activity that is already established as fun. We believe this approach will make visualization more accessible to the general public.

LEGO structures are not only fun to create, but also fun to look at. They can serve as public art while also enabling participants to read patterns in the data in real time, as structures are being constructed. For example, encoding sky color in a row of LEGO over the course of a 3-day festival makes it possible to track weather patterns during the time period of data collection. This has the potential to further engage audiences, even if they do not build LEGO stacks themselves.

\subsection{LEGO are colorful}

Many types of visual features are used to encode information in visualizations, including color, texture, size, and shape [2]. Color, in particular, is highly flexible for communicating concepts through visualizations because people associate a wide variety of meanings with a given color [5,7,9,12,13,16,17]. For example, a particular shade of red, like LEGO red, can be associated with different emotions (e.g., love and anger), institutions (e.g., University of Wisconsin-Madison and US Republican Party), and foods (e.g., strawberries and pizza sauce), to name only a few examples. Evidence suggests that these associations determine how much people like colors $[12,16]$ and how they interpret colors in visualizations $[1,9,13-15]$. Beyond established associations, people can also follow instructions to encode new associations in colors, such as mapping different counties to pairs of colors (e.g., Dayton County to red and white). Thus, people are readily willing to use the same red LEGO brick to encode a wide variety of information while building LEGO structures.

\subsection{LEGO emphasize visual and tactile interactions}

Our approach uses LEGO as manipulatives; concrete materials (e.g., blocks, sticks, or tiles) that are helpful for demonstrating quantitative concepts and aiding in reasoning $[8,10]$. LEGO provide a different way to tap into preferences and attitudes compared with traditional verbal surveys. This may be especially useful for doing research with children or others for whom traditional language-based tools are not well-suited. We believe this offers unique advantages as a tool for finding out about what people think and feel and for presenting that information back to them in an immediate, engaging, and memorable way.

\subsection{LEGO facilitates group discussion}

Answering questions only in color creates rich conversations among people putting their stacks together, which can also be captured by staff. Each person's stack is a visual poem about them. For example, one child said that friendship is purple because it is made of blue and red, just like two friends blending together. This project provides an easy entry point for participants to think about their thinking [4]; why did they make the connections to certain colors? How did they trace in their own minds the origin of their personal color language? Moreover, comparing one's own stack to others stacks can enable empathetic thinking [11].

\subsection{LEGO structures are readily digitized}

We can capture images of LEGO structures and convert them into digital data. Once converted, the data can be plotted and analyzed using traditional methods. Details on how this can be accomplished are described below.

\subsection{LEGO are ubiquitous}

LEGO are relatively easy to acquire, are already distributed in households across the world, and are intuitive to use. These features make the project scalable and self-explanatory without great cost or resources. 


\section{Project 1: "What COLOR IS _-_?"}

Our project, "What Color is _-_--_?" at the 2018 Wisconsin Science Festival, marks the first time that LEGO has been used to conduct a large-scale survey and to store and visualize the findings in real time. Over two 2018 summer pilot tests and four days in October, more than 2,000 children visiting UW-Madison's Discovery Building participated avidly in our project. We asked them to answer 21 questions about themselves and the world using only $2 \times 4$ LEGO blocks in 12 colors. Each child's "answers" in this LEGO language formed a vertical stack 21 blocks high. Figure 1 shows some of those stacks combined together into mini walls in the window of the Discovery Building at the University of Wisconsin-Madison in Madison, Wisconsin, USA.

Kids, along with their families, friends, and teachers often sat for 15 or 20 minutes discussing their responses and building their stacks, before putting them in the many window racks design by Discovery Outreach staff. The project ultimately filled a dozen of the Discovery Building's windows, which drew attention from visitors inside and out, bringing in additional traffic.

\subsection{LEGO survey questions}

Using the template designed by Discovery Outreach staff (Figure 2A), we asked each visitor 21 questions, all of the form "What color block do the words below make you think of?" The questions vary from more concrete at the bottom to more abstract at the top. Within this list there are four types of questions:

- Questions 1-4 ask you to encode information according to simple system that we provide. E.g., If you are age 0-5, put in a red block.

- Questions 5, 6,7 and 12 ask you to make observations about the world. E.g., What color is the sky right now? or What color is your shirt?

- Questions 8,9 and 10 ask you about your personal preferences. E.g., What color is your favorite, song, color, or food?

- Questions 11, and 13-21 ask you to make abstract/poetic associations to colors. E.g., What color is justice? Hope? Anger?

\subsection{Storing, manipulating, and analyzing data}

We digitized data stored in LEGO structures by capturing images a phone camera or flatbed scanner, detecting the colors, and translating them into color words stored in tables.

While detecting colors of LEGO is a process that has been studied in the field of robotics $[6,18]$, our goal was to translate this information into a more traditional format for data analysis. The process to convert from images to spreadsheets was accomplished using a custom written project utilizing the OpenCV framework [3]. As photos of the blocks also contained other visual information (Figure 2B), the program first found the boundaries of the LEGO stacks. From this, a warp matrix was used to project the LEGO images onto an axis-aligned grid image. Color samples were taken from the center of each grid cell and compared against human derived labels to find the closest approximate LEGO color. The results of these operations were exported to a commaseparated-value (CSV) file which could be used for data analysis and $3 \mathrm{D}$ visualizations [6] (Figure 2C).

Thus, we go from a picture of wall consisting of 10 peoples responses (Figure 2B) to an excel spreadsheet that records color names in columns and rows (Figure 2C). This format allows data to be manipulated and analyzed in conventional ways. The developed software and source is available for others to use and explore on github ${ }^{1}$.

The team also built an online version of the project which allows participants to answer the survey and assemble their stacks using photos of blocks rather than actual blocks. While the created software is still in development, the team is working to refine user interface and underlying algorithms to make a better and more robust user experience.

${ }^{1}$ https://github.com/kevinponto/LegoColor2CSV
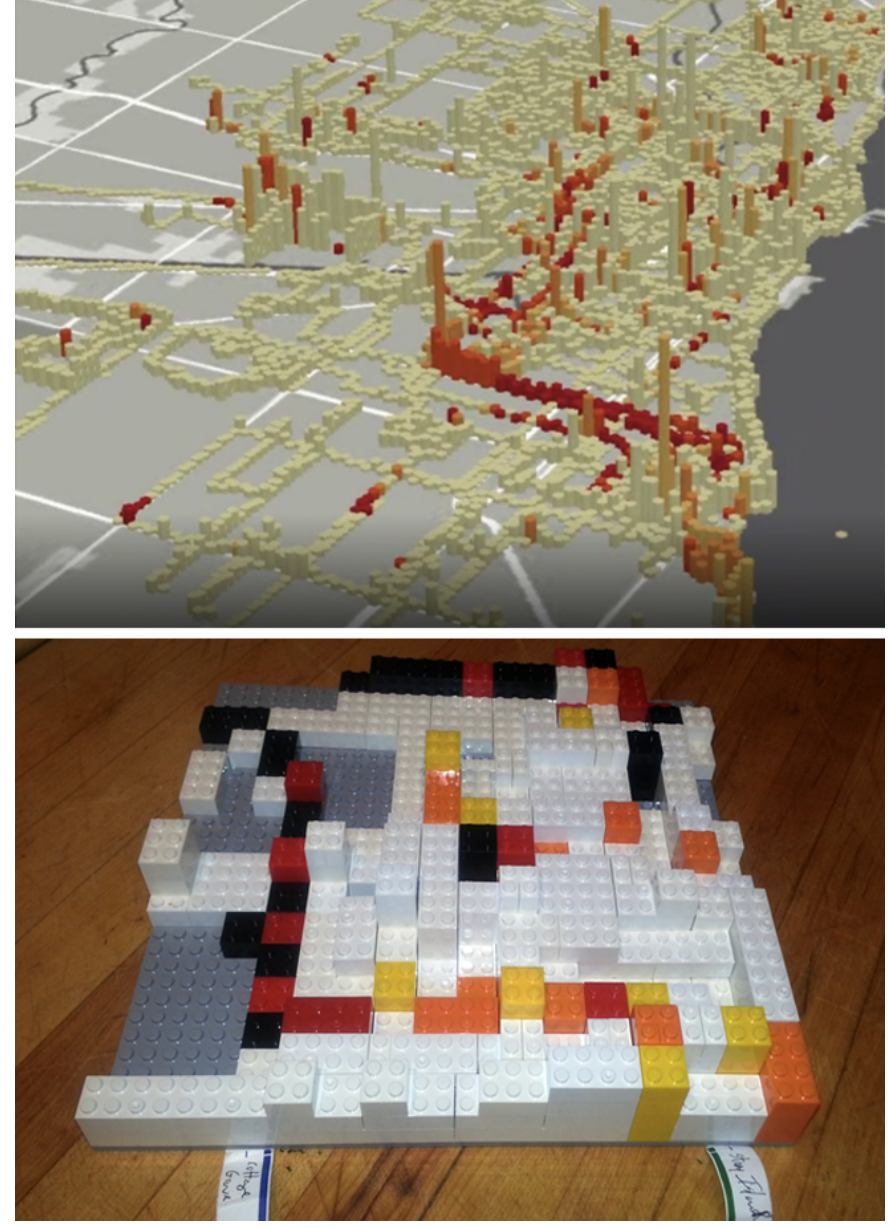

Figure 3. Screen shot from airqualitychicago.org (top) and LEGO model of detail of data from the South East Side of Chicago (bottom) The height of the column represents the amount of data and the color represents air quality (redder, darker colors map to poorer air quality.

\subsection{A new way to explore thoughts and feelings}

We observed high levels of enthusiasm and engagement for making LEGO stacks at our events. Interestingly, the more poetic questions not only entertained participants (both the adults and the children), but also provoked unique reflection on topics like Justice or Friendship.

The language of LEGO is much richer than we could fully explore in this single project. There are many communication modalities that we have yet to study, such as allowing participants to combine multiple smaller blocks to make a blended color for their $2 \times 4$ blocks. For example, if the sky was part blue, white, and gray, they could have made a more nuanced composite block using smaller blocks $(1 \times 4$, $1 \times 1,2 \times 2 \ldots$ etc.). Participants might also use composites for a shirt or for food that have multiple colors. Many kids actually asked questions like, "my favorite food is pizza, which is white and red, which color should I pick?" Similarly for abstract concepts, some felt that "justice" should be "half black and half white."

\section{Citizen Science and Data Visualization}

In related work, Stuart Flack and his colleagues at the Environmental Law \& Policy Center http://elpc.org/ and the Invisible Institute https://invisible.institute/introduction are working with community groups in Chicago to measure harmful air born particulate matter (PM 2.5) with small monitors that clip to a belt loop or back pack. High levels of PM 2.5 typically result from gas and diesel engines and are especially dangerous to people with asthma and other respiratory illness. Many troubled neighborhoods on Chicago's South and Westside show 
elevated levels of both PM 2.5 and of asthma.

Members of the community are typically high school and college students who are part of a summer program designed to provide STEM ed and advocacy experience. They carry the monitors around the neighborhood and the data are sent directly to a cloud-based public site (airqualitychicago.org) where its analyzed and mapped. Fig 3 shows a ArcGIS depiction of some of our community data as it appears on the site. The height of the column shows the amount of data and color depth (beige red) shows the danger levels of PM 2.5.

While this is a certainly a good example of timely data collection and visualization using cloud based ArcGIS, our experience is that much more needs to be done to make this information memorable, important, and actionable for people in the community. Enter LEGO. This years work will not only include data gathering, it will also include community workshops where folks will take their data and translate it into LEGO maps.

The construction in 3 zooms in on a 2 mile square on the South east side of Chicago. We see more detail about a stretch of high readings in the first map (the red horizontal line at the bottom of the first map). As before, the height of the column indicates the amount of data gathered and color indicates severity of PM 2.5 in the following order from safe to severe: white-yellow-orange-red-black.

With the guidance of a moderator who also understands the underlying science of PM 2.5 and data analytics, teams of 2-4 community members (who have likely gathered the data also) will be able to put LEGO displays like these together in 30 minutes. LEGO structures can then be displayed in community centers around the city and used to spark discussions in neighborhood meetings. An added benefit of using LEGO is that community members can easily add more LEGO as more data are collected. We also believe that LEGO maps will be useful in our neighborhood based advocacy efforts.

Instead of seeing yet another picture on a computer, receiving a printed report, or hearing a lecture by an expert, community members are participating in interactive experiences. Collecting data with our handheld monitors is fun and engaging, and assembling and examining that data is memorable and illuminating.

In addition to working with air quality data, we will explore how to use this LEGO technique to visualize other community data (e.g., asthma attack incidence, construction, pot holes, walking routes, and police coverage). We anticipate weaving this technique into a variety of presentations, workshops, forums, advocacy meetings, and perhaps even theatrical performances.

\section{ConClusion}

Broadly, we believe that LEGO_-and perhaps other uniform, cheap, manipulable, multi-colored objects (e.g. ping pong balls, screws, nuts, bolts, rice, beans, lentils...etc.) — can be used to create effective and engaging real time visualization for a variety data and settings. For example, LEGO is well suited to some of the common geo spatial mapping visualization commonly done today with Arc GIS software, as we have shown in our air quality example. It can do some of the visualizations we typically associate with software like Excel and Tableau. As both of our projects show, LEGO facilitates new kinds of visualization that are unique to LEGO-based approaches.

Using LEGO for visualization may offer creative new ways to engage the public with data and analysis on important science and policy topics. Through that engagement, we see great potential for more coherent public discussion and more political support on a host of data-driven issues, such as climate changes, air quality, economic opportunity, and racial inequality.

\section{ACKNOWLEDGMENTS}

We thank the following team members for their invaluable help on this work: Kiana Courtney, Tiffany Werner, Lucas Stephens, Susan Mudd, David Knight, Henry Shah, Jamie Kalvin, Laura Heisler, Val Blair, Shauna Baranczyk, Jo Withers, Dan Murphy, and Yuke Liang.

\section{REFERENCES}

[1] L. Bartram, A. Patra, and M. Stone. Affective color in visualization. In Proceedings of the 2017 CHI Conference on Human Factors in Computing Systems, pages 1364-1374. ACM, 2017.

[2] J. Bertin, W. J. Berg, and H. Wainer. Semiology of graphics: diagrams, networks, maps, volume 1. University of Wisconsin press Madison, 1983.

[3] G. Bradski and A. Kaehler. Learning OpenCV: Computer vision with the OpenCV library. " O'Reilly Media, Inc.", 2008.

[4] N. R. Council et al. How people learn: Brain, mind, experience, and school: Expanded edition. National Academies Press, 2000.

[5] A. J. Elliot and M. A. Maier. Color-in-context theory. In Advances in experimental social psychology, volume 45, pages 61-125. Elsevier, 2012.

[6] T. Engelke, S. Webel, and N. Gavish. Generating vision based lego augmented reality training and evaluation systems. In 2010 IEEE International Symposium on Mixed and Augmented Reality, pages 223-224. IEEE, 2010.

[7] N. Humphrey. The colour currency of nature. Colour for architecture, 5:95-98, 1976.

[8] E. V. Laski, J. R. Jordan, C. Daoust, and A. K. Murray. What makes mathematics manipulatives effective? lessons from cognitive science and montessori education. SAGE Open, 5(2):2158244015589588, 2015.

[9] S. Lin, J. Fortuna, C. Kulkarni, M. Stone, and J. Heer. Selecting semantically-resonant colors for data visualization. In Computer Graphics Forum, volume 32, pages 401-410. Wiley Online Library, 2013.

[10] S. C. Marley and K. J. Carbonneau. Theoretical perspectives and empirical evidence relevant to classroom instruction with manipulatives, 2014.

[11] J. Mezirow. Learning as Transformation: Critical Perspectives on a Theory in Progress. The Jossey-Bass Higher and Adult Education Series. ERIC, 2000.

[12] S. E. Palmer and K. B. Schloss. An ecological valence theory of human color preference. Proceedings of the National Academy of Sciences, 107(19):8877-8882, 2010.

[13] K. B. Schloss. A color inference framework. In . G. V. P. L. MacDonald, C. P. Biggam, editor, Progress in Colour Studies: Cognition, language, and beyond. John Benjamins, Amsterdam, 2018.

[14] K. B. Schloss, C. C. Gramazio, A. T. Silverman, M. L. Parker, and A. S. Wang. Mapping color to meaning in colormap data visualizations. IEEE transactions on visualization and computer graphics, 25(1):810-819, 2019.

[15] K. B. Schloss, L. Lessard, C. S. Walmsley, and K. Foley. Color inference in visual communication: the meaning of colors in recycling. Cognitive research: principles and implications, 3(1):5, 2018.

[16] K. B. Schloss and S. E. Palmer. An ecological framework for temporal and individual differences in color preferences. Vision research, 141:95-108, 2017.

[17] V. Setlur and M. C. Stone. A linguistic approach to categorical color assignment for data visualization. IEEE transactions on visualization and computer graphics, 22(1):698-707, 2016.

[18] S. Zhao, W. Tan, S. Wen, and C. Guo. Research on robotic education based on lego bricks. In 2008 International Conference on Computer Science and Software Engineering, volume 5, pages 733-736. IEEE, 2008. 